\title{
Parameter Study of Sound Propagation between City Canyons with Coupled FDTD- PE Model
}

\author{
Timothy Van Renterghem, Dick Botteldooren \\ Ghent University, Department of Information Technology, Sint-Pietersnieuwstraat 41, B- 9000 Gent, Belgium, \\ \{Timothy.Van.Renterghem,Dick.Botteldooren\}@intec.Ugent.be, \\ Erik Salomons \\ TNO TPD, P.O. Box 155, 2600 AD Delft, The Netherlands, Erik.Salomons@tno.nl
}

\begin{abstract}
In this paper, detailed numerical simulations of sound propagation in an urban environment are presented. The focus is on propagation from a "source canyon" to a distant "receiver canyon". Such numerical simulations have to take into account multiple reflections, both specular and diffuse, multiple diffractions, the presence of different building materials, and also the interaction with a moving atmosphere. The finite-difference time-domain (FDTD) technique, solving the moving inhomogeneous medium sound propagation equations, can be used in such an environment. In contrast to other models, very accurate simulations can be performed since the interaction between the different effects involved is accounted for. A coupled FDTD-PE model is applied to improve computational efficiency. The problem size is further decreased by assuming that the geometry of the source and receiver canyon is identical. Parameters like canyon geometry, diffusivity of the façades and wind speed are investigated.
\end{abstract}

\section{Introduction}

Common noise reducing measures like noise barriers are difficult to apply in city centres. Therefore, a good city planning is of major importance. A good knowledge of the factors that influence sound propagation is needed.

It was observed that easy access to a quiet place in a noisy area reduces the percentage highly annoyed residents [1]. The preservation of silent places in a city (e.g. backyards) can therefore help to reduce the urban noise problem [2].

The centres of large cities can be seen as an ensemble of so-called "city canyons". Accurate calculations of sound propagation from a source canyon (e.g. a street) towards a shielded receiver canyon is of interest for quiet side conservation. Traditional models [3] tend to overestimate shielding. Recently, the Equivalent Sources Method (ESM) [4] was used successfully to model sound propagation between city canyons.

ESM and similar methods that are based essentially on the Green's function can not be used when the sound propagation medium is moving and/or inhomogeneous. Therefore, the finite-difference time-domain (FDTD) method, solving the moving inhomogeneous medium sound propagation equations [5-7] is interesting in this view.

The computational effort required for FDTD simulations is large. In this paper, this disadvantage is (partly) overcome by using a coupled 2D FDTD-PE model, which has shown to drastically limit computing time and memory usage [8]. Calculation times are further reduced by assuming that the source and receiver canyon are geometrically identical.

\section{Calculation method}

Figure 1 shows the setup for the calculations in this paper. In the source canyon, FDTD calculations are performed. Staggered-in-space calculations are combined with the prediction-step staggered-in-time (PSIT) approach to evaluate the moving-medium sound propagation equations [6]. Perfectly matched layers are used to simulate an unbounded atmosphere. At short distance from the canyon edge, time signals are recorded on a vertical array to generate starting functions for the Parabolic Equation (PE) method. Next, a transition from the time domain to the frequency domain is performed by means of FFT. Finally, the Green's Function PE (see e.g. Ref. [9]) method is used to calculate sound propagation up to a receiver at the symmetry plane, for a number of frequencies. The latter uses the effective sound speed approach. Details on the coupling between FDTD and $\mathrm{PE}$ can be found in Ref. [8].

Symmetry (see Figure 1) and reciprocity allows approximating the transfer function from the source to the receiver by two times the transfer function from the source towards a point on the symmetry plane, at a limited height. Using this approach, reflection on the rigid roof is counted twice, so $6 \mathrm{~dB}$ must be subtracted from the doubled sound pressure levels at the symmetry plane.

Calculations with this coupled model, exploiting symmetry, were shown to be in very good agreement with the FDTD method applied to the complete sound propagation region. 


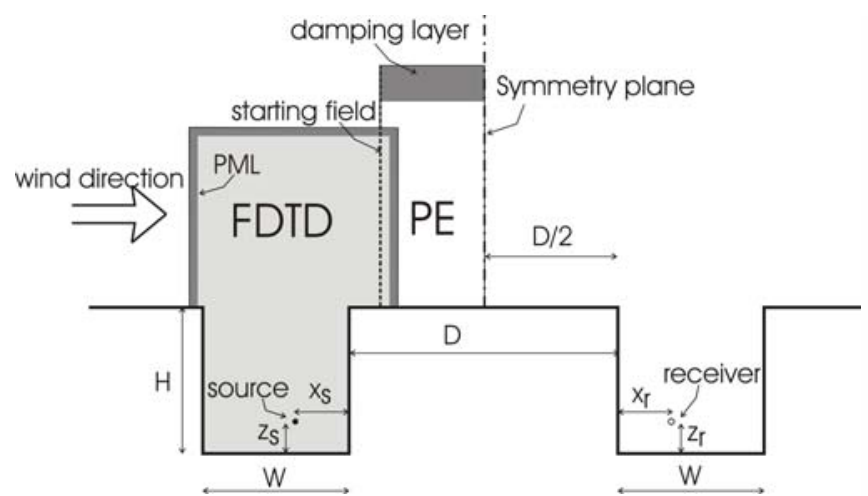

Figure 1. Setup of coupled FDTD-PE model used for the parameter study, exploiting geometrical symmetry

Two-dimensional simulations are performed. This means that infinitely long source and receiver canyons are modelled, while the (line) sources are infinitely long and coherent. Effects of the coherent line source are partly averaged out when results are expressed in $1 / 3$ octave bands.

\section{Parameter study}

Unless explicitly investigated, the following parameters were used in the simulations. The building height $(\mathrm{H})$ and canyon width $(\mathrm{W})$ equal $10 \mathrm{~m}$. The distance between the canyons (D) is $100 \mathrm{~m}$. The source and receiver are placed at equal distance from the symmetry plane. Source and receiver height $\left(\mathrm{z}_{\mathrm{s}}=\mathrm{z}_{\mathrm{r}}\right)$ are $1 \mathrm{~m}$, the distance towards the middle building $\left(\mathrm{x}_{\mathrm{s}}=\mathrm{X}_{\mathrm{r}}\right)$ is $4 \mathrm{~m}$. The roofs and street coverage are rigid. The façades have a normalized frequency-independent real impedance of 10 . Results are expressed relative to free field calculations, in $1 / 3$ octave bands. In the PE region, 20 frequencies were simulated per frequency band. Unless otherwise stated, the sound propagation medium is homogeneous and non-moving.

\subsection{Canyon width}

The effect of canyon width is shown in Figure 2. The shielding increases with increasing frequency. For very low frequencies, almost no shielding is observed. The shielding of very narrow canyons is large. With increasing canyon width, the relative sound pressure levels in the receiver canyon increase. When the width of the canyon exceeds its height (starting from $\mathrm{W}=10$ $\mathrm{m}$ in our example), the sound pressure levels become more or less constant.

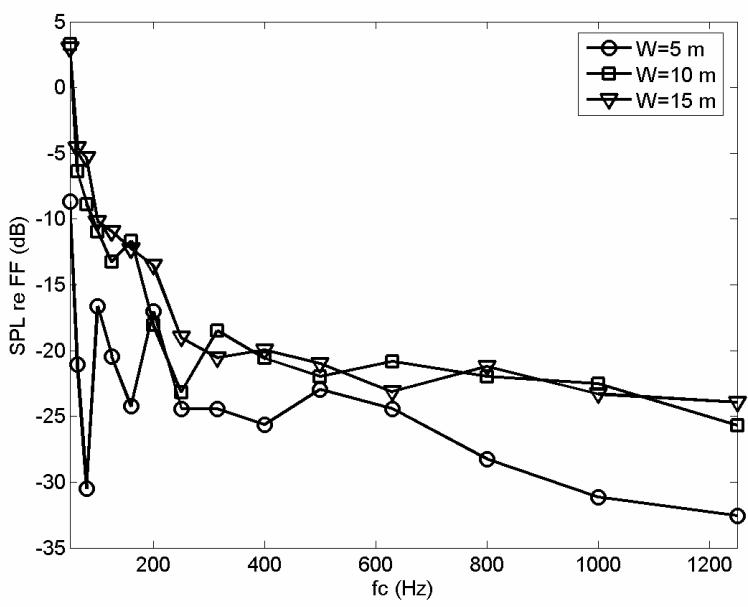

Figure 2. Sound pressure levels relative to free field, in $1 / 3$ octave bands, for different widths of the canyons, in a non-moving atmosphere. Façades reflect purely specularly.

\subsection{Moving atmosphere}

The flow field near the canyon geometry is calculated with CFD software Fluent [10]. A k- $\varepsilon$ turbulence model is used to account for the large, turbulent motions. A logarithmic inflow profile $u(z)=\left(u_{*} / \kappa\right) \ln \left(1+z / z_{0}\right)$ is used, where $u_{*}$ is the friction velocity, $\kappa$ is the von Kármán constant $(\kappa=0.4)$, and $z_{0}$ is the ground roughness length. This profile corresponds to downwind sound propagation from the source canyon to the receiver canyon. A simulation is performed with $u_{*}=0.5 \mathrm{~m} / \mathrm{s}$ and $1 \mathrm{~m} / \mathrm{s}$, while $z_{0}=0.5$ $\mathrm{m}$. The flow field near the source canyon is shown in Figure 3.

It can be seen from Figure 4 that the wind effect is large. The downwind refraction encountered in open field is enhanced by the so-called building-induced refraction of sound, which is more or less similar to the screen-induced refraction of sound by wind [6, 11]. Downwind refraction becomes more pronounced with increasing frequency and with increasing wind speed. A decrease in shielding compared to a non-moving atmosphere of about $10 \mathrm{~dB}$ is observed when $u_{*}=1$ $\mathrm{m} / \mathrm{s}$, for the frequency bands that are important for traffic noise. It is clear that the effect of wind can not be neglected in these situations. 

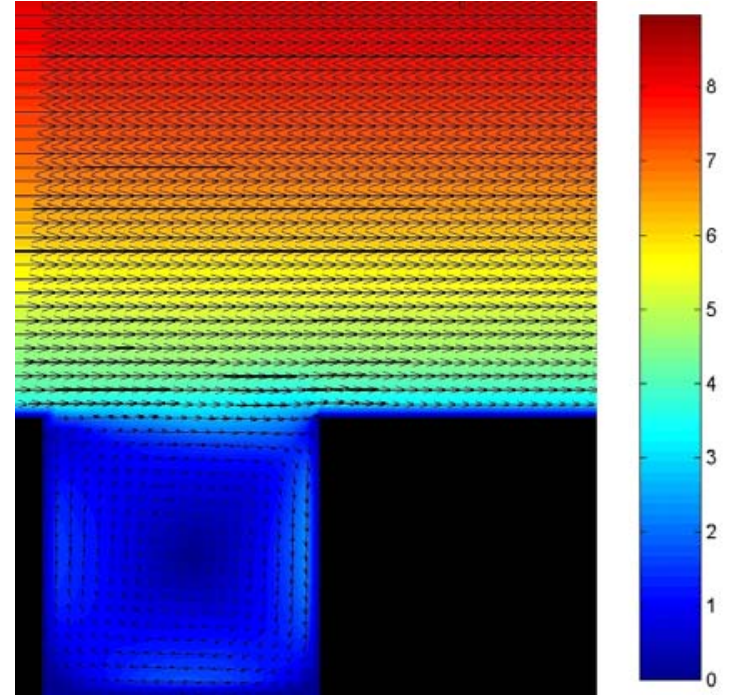

Figure 3. Flow field near source canyon. The magnitude of the flow velocity is indicated in $\mathrm{m} / \mathrm{s}$. The vectors indicate the flow direction.

The wind field above a city is usually highly turbulent. A simulation, including both refraction and turbulence is performed. Turbulent scattering is accounted for during the PE calculations only. More information on the latter can be found in Ref. [9]. A Von Kármán turbulence spectrum is used, with a structure velocity parameter $\mathrm{C}_{\mathrm{v}}{ }^{2}$ equal to $10 \mathrm{~m}^{4 / 3} / \mathrm{s}^{2}$. The correlation length was taken to be $10 \mathrm{~m}$. Temperature turbulence is not accounted for. It can be seen in Figure 4 that the wind effect increases slightly when the turbulent atmosphere is accounted for. The main effect of the wind is however caused by downwind refraction.

\subsection{Diffuse reflection}

The importance of diffuse reflection in urban environments is well-known. A commonly used method to account for diffuse reflection is assuming that a certain amount of energy is transferred from the specular (coherent) field to the diffuse field with each reflection. The scattering coefficient quantifies this transfer. This approach assumes that façades scatter randomly from every point [13]. In reality however, diffusing elements are well-localized. In the FDTD simulation, diffuse reflection is explicitly modelled by making surfaces irregular. Recesses and protrusions due to windows and window sills are modelled near the façade, together with a rough wall, as shown in detail in Figure 5. The windows itself and the window sills are modelled as rigid, while the rest of the façade has a normalized real impedance of 10 .

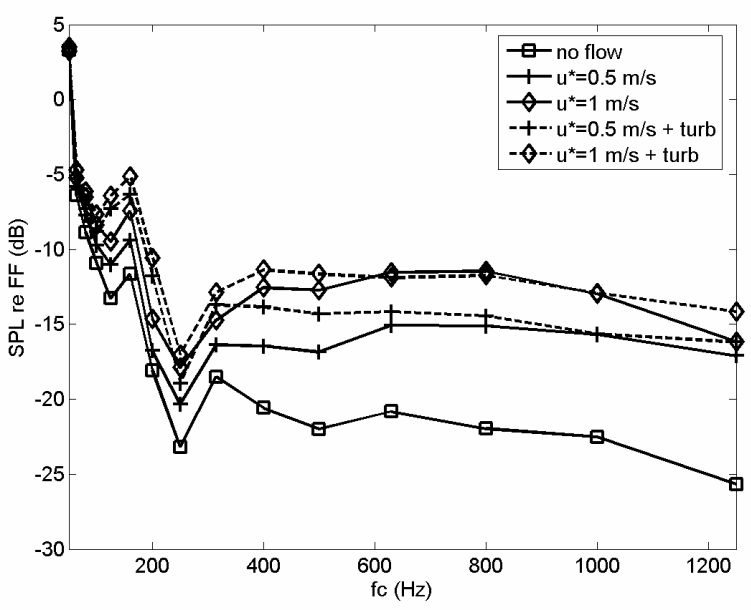

Figure 4. Sound pressure levels relative to free field, in $1 / 3$ octave bands, in a non-moving, in a refracting nonturbulent and in a refracting turbulent atmosphere. In

case of a refracting atmosphere, two wind speed profiles are used. Façades are flat. The canyon width is $10 \mathrm{~m}$.

In figure 6 , the effect of such a profiled façade is compared to a completely flat façade, with the same impedances, for sound propagation from the source to the receiver canyon. The positive effect of diffuse reflection is important and increases with frequency in our example.

It needs to be mentioned that scattering is only modelled in upward and downward direction, since 2D simulations are performed

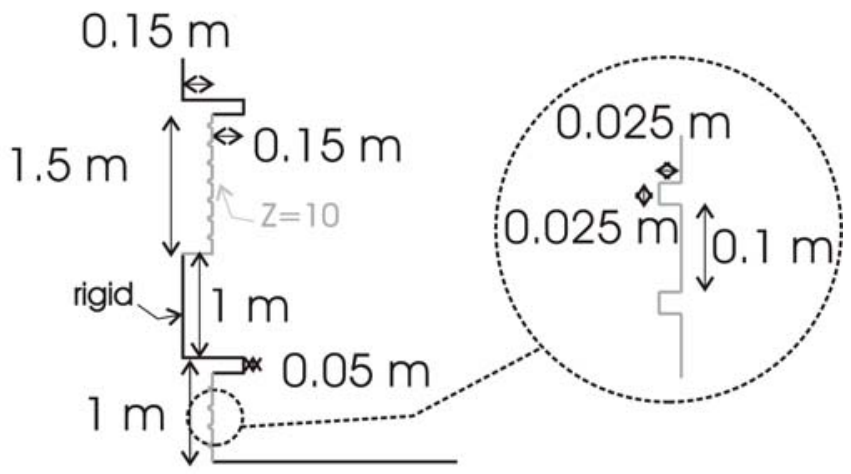

Figure 5. Part of the diffusely reflecting façade that is modelled. 


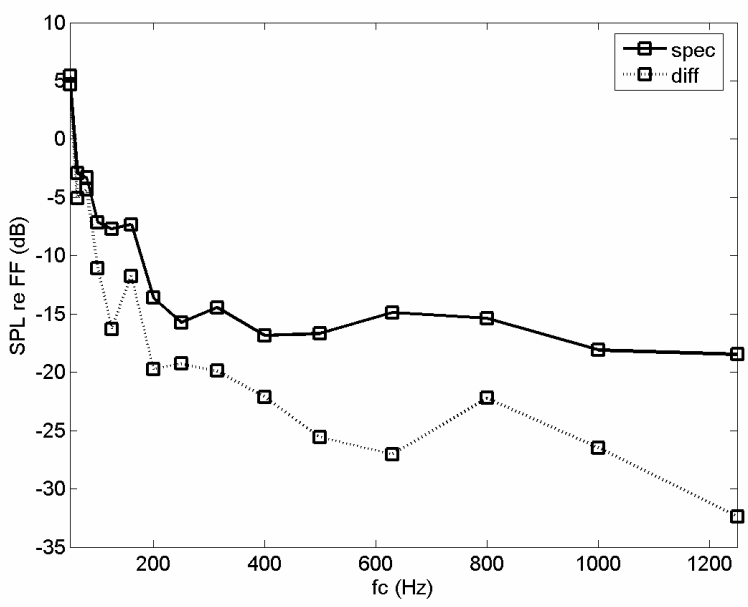

Figure 6. Relative sound pressure levels relative to free field, in 1/3 octave bands, in a non-moving atmosphere. The (partly) diffusely reflecting façade shown in Figure 5 is compared to an equivalent flat façade with the same impedances. The canyon width is $10 \mathrm{~m}$.

\section{$4 \quad$ Summary}

In this paper, 2D simulations of sound propagation from a source to a distant receiver canyon are performed. Canyon width, the effects of a moving atmosphere (refraction + turbulent scattering) and the effect of diffuse reflection are investigated in detail. An important conclusion is that refraction by wind and diffuse reflection are important and therefore need to be taken into account when accurately predicting sound levels in highly shielded urban areas.

\section{References}

[1] E. Ohrstrom, 'Psycho-social effects of traffic noise exposure’, J. Sound Vibr., Vol. 151, pp. 513-517 (1991).

[2] T. Kihlman, 'Quiet side and high façade insulation - means to solve the city noise problem', Proc. Internoise 2001, The Hague, The Netherlands (2001).

[3] T. Kihlman, M. Ögren, W. Kropp, 'Prediction of urban traffic noise in shielded courtyards', Proc. Internoise 2002, Dearborn, MI, USA.

[4] M. Ögren, W. Kropp, 'Road traffic noise propagation between two dimensional city canyons using an equivalent sources approach', Acta Acustica united with Acustica, Vol. 90, pp. 293-300 (2004).

[5] R. Blumrich, D. Heimann, 'A linearized Eulerian sound propagation model for studies of complex meteorological effects', J. Acoust. Soc. Am. , Vol. 112, pp. 446-455 (2002).

[6] T. Van Renterghem, D. Botteldooren, 'Numerical simulation of the effect of trees on downwind noise barrier performance', Acta Acustica united with Acustica, Vol. 89, pp. 764-778 (2003).

[7] V. Ostashev, D. Wilson, L. Liu, D. Aldridge, N. Symons, D. Marlin, 'Equations for finitedifference, time-domain simulation of sound propagation in moving inhomogeneous media and numerical implementation', J. Acoust. Soc. Am., Vol. 117, pp. 503-517 (2005).

[8] T. Van Renterghem, E. Salomons, D. Botteldooren, 'Efficient FDTD-PE model for sound propagation in situations with complex obstacles and wind profiles', Acta Acustica united with Acustica, Vol. 91, pp. X-X (2005).

[9] E. Salomons, 'Computational atmospheric acoustics’, Kluwer, Dordrecht (2001).

[10]Fluent, Computational Fluid Dynamics Software, version 6, Fluent, Incorporated, Centerra Resource Park, 10 Cavendish Court, Lebanon, NH 03766.

[11]E. Salomons, 'Reduction of the performance of a noise screen due to screen-induced wind-speed gradients. Numerical computations and wind tunnel experiments', J. Acoust. Soc. Am., Vol. 105, pp. 2287-2293 (1999).

[12] M. Ismail, D. Oldham, 'A scale model investigation of sound reflection from building façades', Applied Acoustics, Vol. 66, pp. 123-147 (2005). 


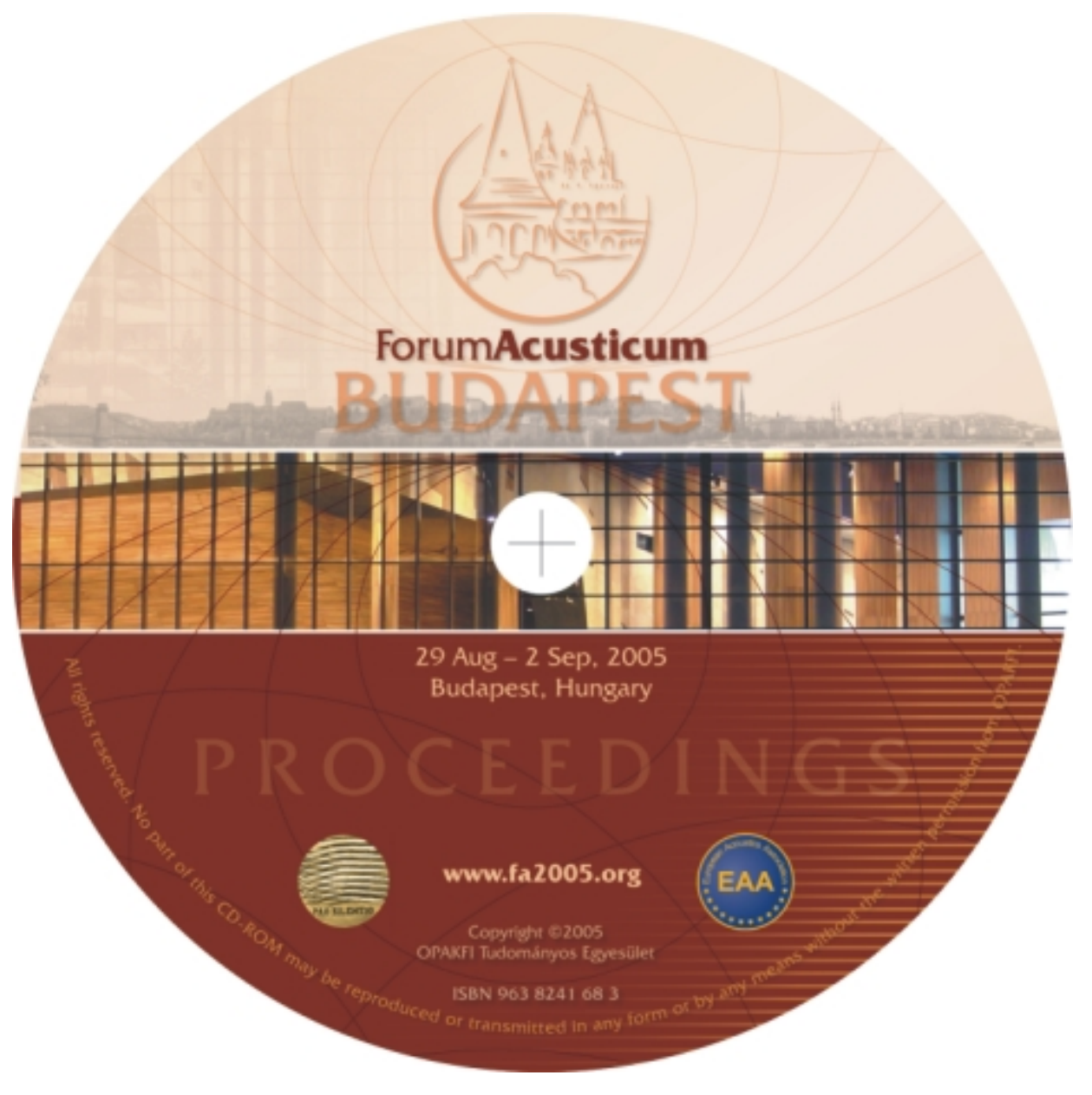

